\title{
Erratum: Clinical Usefulness of Uric Acid as a Biomarker for Knee Osteoarthritis: A Comparative Analysis With Plain Radiography and Musculoskeletal Ultrasound
}

\author{
Seong-Kyu Kim, M.D., Ph.D., Ui Hong Jung, M.D., Jung-Yoon Choe, M.D., Ph.D. \\ Division of Rheumatology, Department of Internal Medicine, Daegu Catholic University School of Medicine, Daegu, Korea
}

J Rheum Dis 2020;27:51-60

https://doi.org/10.4078/jrd.2020.27.1.51

The original version of this article contained a typographical error in the Abstract, Main text, and Figure 1 (the corrected contents are displayed in underline). These errors have now been corrected in the online version of the Article.

\section{In the 'Abstract' section (p. 51):}

'Ultrasonographic abnormalities in knee OA includedsynovial hypertrophy, suprapatellar effusion, cartilage degradation, and osteophyte formation.' should be replaced with

'Ultrasonographic abnormalities in knee OA included synovial hypertrophy, suprapatellar effusion, cartilage degradation, and osteophyte formation'

\section{In the second paragraph of 'Results' section (p. 54):}

'Serum uric acid, urine uric acid, and serum urine/creatinine ratio among three groups were not statistically different, and only urine urine/creatinine ratio was significantly higher in K-L grade III than grade II (0.83 [0.55 1.02] vs. 0.59 [0.46 0.67], $\mathrm{p}=0.043)$.' should be replaced with

'Serum uric acid, urine uric acid, and serum urine/creatinine ratio among three groups were not statistically different, and only urine uric acid/creatinine ratio was significantly higher in K-L grade III than grade II $(0.83$ [0.55 1.02] vs. 0.59 [0.46 0.67], $\mathrm{p}=0.043)$.'

\section{In the fourth paragraph of 'Results' section (p. 56):}

'In contrast, spot urine uric acid and uric acid/creatinine ratio in patients with synovial hypertrophy were marginally lower in patients with synovial hypertrophy compared to those without ( $\mathrm{p}=0.055$ for both).' should be replaced with

'In contrast, spot urine uric acid and uric acid/creatinine ratio were marginally lower in patients with synovial hypertrophy compared to those without ( $\mathrm{p}=0.055$ for both)'.

\footnotetext{
Corresponding to : Seong-Kyu Kim (D) http://orcid.org/0000-0002-7780-0167

Division of Rheumatology, Department of Internal Medicine, Daegu Catholic University School of Medicine, 33 Duryugongwon-ro 17-gil, Nam-gu, Daegu 42472, Korea. E-mail : kimsk714@cu.ac.kr
} 
4. The p-value of urine uric acid $(\mathrm{mg} / \mathrm{dL})$, in Figure 1:

' $p>0.021$ ' should be replaced with ' $p=0.021$ ' (the corrected Figure is reproduced here).
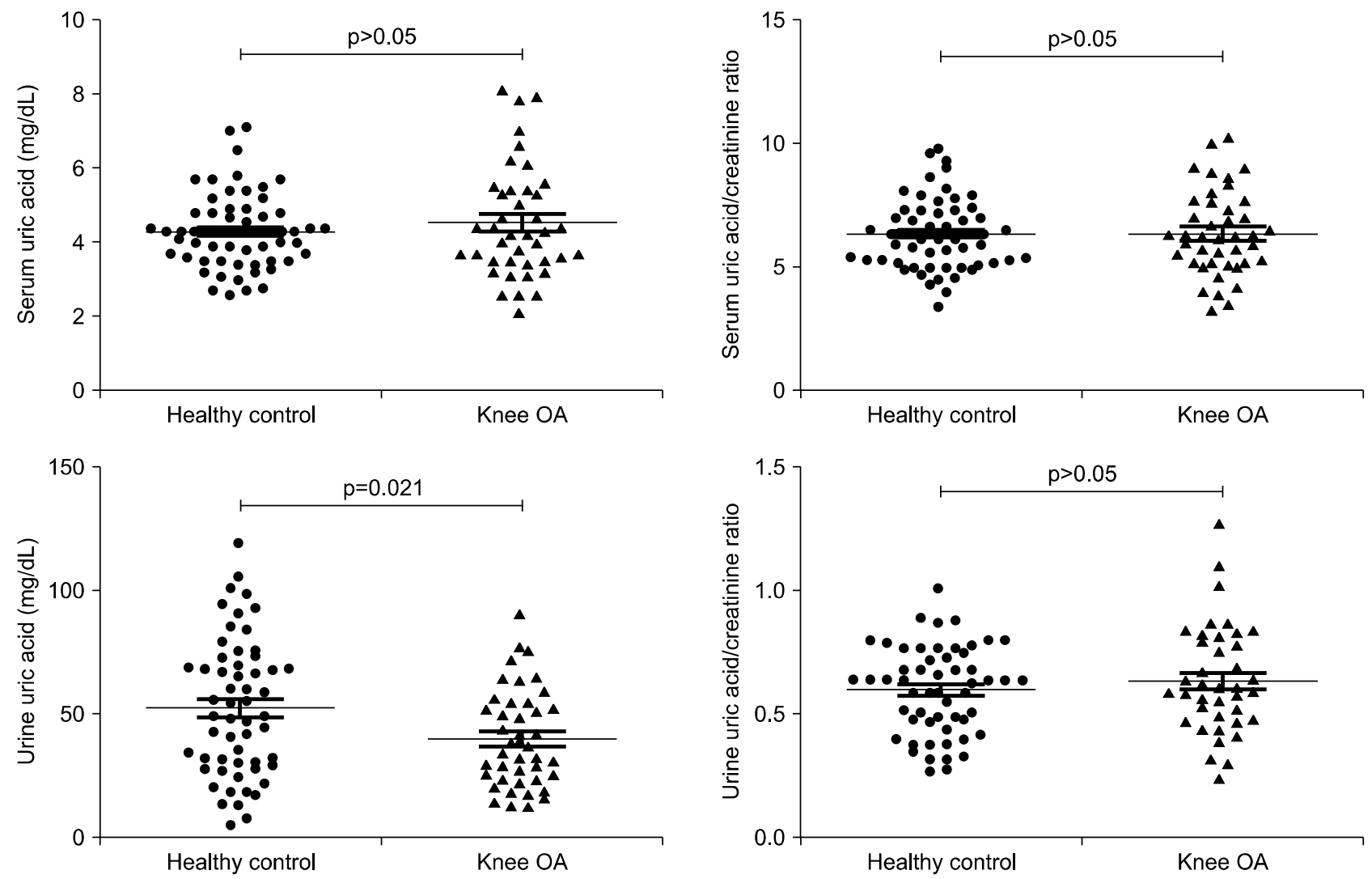

Figure 1. Comparison of uric acid and uric acid/creatinine ratio in serum and urine between patients with knee osteoarthritis and healthy controls. OA: osteoarthritis.

We apologize for our mistake and any inconvenience this may have caused. 